\title{
Effects of 4 weeks of Lactobacillus plantarum 299v supplementation on nutritional status, enteral nutrition tolerance, and quality of life in cancer patients receiving home enteral nutrition - a double- blind, randomized, and placebo-controlled trial
}

Kaźmierczak-Siedlecka Karolina ( $\square$ leokadia@gumed.edu.pl )

Gdanski Uniwersytet Medyczny https://orcid.org/0000-0002-0283-1436

\author{
Marcin Folwarski \\ Medical University of Gdańsk \\ Jakub Ruszkowski \\ Medical University of Gdańsk \\ Karolina Skonieczna-Żydecka \\ Pomeranian Medical University in Szczecin \\ Waldemar Szafrański \\ Nutritional Counselling Centre in Gdańsk \\ Wojciech Makarewicz \\ Medical University of Gdańsk
}

\section{Research}

Keywords: Lactobacillus plantarum 299v, home enteral nutrition, cancer, nutritional status, quality of life

Posted Date: April 20th, 2020

DOI: https://doi.org/10.21203/rs.3.rs-22190/v1

License: (9) This work is licensed under a Creative Commons Attribution 4.0 International License.

Read Full License 


\section{Abstract}

\section{Background}

Several human trials have confirmed that Lactobacillus plantarum 299v (Lp299v) relief the gastrointestinal symptoms observed in patients with irritable bowel syndrome, such as nausea, vomiting, and diarrhoea. These symptoms are similar to those associated with home enteral nutrition and they affect nutritional status as well as patients' quality of life.

\section{Methods}

The current double-blind, randomized, and placebo-controlled study included 35 cancer patients receiving home enteral nutrition. There were 2 groups of participants consuming either $2 \times 10^{10}$ CFU of Lp299v $(n=$ $21)$ or placebo $(n=14)$ for 4 weeks. The aims of this study were to determine the effect of Lp299v on nutritional status, enteral formula tolerance, and quality of life in cancer patients.

Results

An increase in serum albumin concentration was significantly higher in the Lp299v group than in the placebo group at the endpoint $(p=0.032)$. Moreover, the changes in the frequency of vomiting and flatulence were significantly reduced at week 4 compared to baseline in the Lp299v group $(p=0.0117)$. The improvement of quality of life was observed in both groups; however, with no statistically significant differences between the analysed groups $(p>0.05)$.

\section{Conclusions}

We have demonstrated that administration of Lp299v in cancer patients receiving home enteral nutrition may improve laboratory parameters, predominantly the concentration of albumin, however, overall it does not have an impact on nutritional status. Lp299v may reduce the gastrointestinal symptoms related to enteral nutrition; notwithstanding, the improvement of quality of life may be the result of enteral nutrition rather than the effect of administration of Lp299v.

Trial registration

ClinicalTrials.gov (identifier: NCT03940768)

\section{Background}

Nutritional treatment is an important component of multidisciplinary anti-cancer therapy. According to many trials, an appropriate nutritional support reduces the side effects of anti-cancer treatment, shortens the length of hospital stay, improves nutritional status and the clinical outcome as well as patients' quality of life (QOL) (1). Home enteral nutrition (HEN) is recommended for patients with efficiently functioning gastrointestinal tract who do not require hospitalization [1]. Accordingly to Villar Taibo et al., 
as many as $75 \%$ of patients qualified for HEN are malnourished [2]. Furthermore, several trials confirmed that HEN positively affects the patients' nutritional status presenting multiple benefits; however, enteral nutrition (EN) may also be associated with some complications, such as nausea, vomiting, flatulence as well as abdominal pain [3]. Notwithstanding, diarrhoea is the most prevalent side effect during HEN. It has an impact on overall recovery in the postoperative period, causes fluid and electrolyte loss, prolongs the length of hospital stay as well as increases mortality and morbidity [3]. The incidence of diarrhoea in patients receiving EN varies between 12 and $68 \%$ patients $[4,5]$. However, the pathogenesis of this symptom involves several factors. It is mainly caused by the unadjusted speed of the enteral formula administration and gastrointestinal tract's reaction to substances with higher osmolarity. In cancer patients, diarrhoea may be an adverse event during anti-cancer therapy (e.g. chemotherapy- or radiotherapy-related diarrhoea). Another hypothesis is that diarrhoea might be caused by changes in the gut microbiota [3,6]. Overall, the inadequate composition of gut microbiota has been linked to poor eating habits (high-fat diet, low fiber intake), administration of antibiotics, side effects of anti-cancer therapy, and surgical procedures [7-9]. In cancer patients the increased abundance of several specific bacteria was noted; for instance, the high counts of Fusobacterium nucleatum, Bacteroides fragilis, Streptococcus bovis, Peptostreptococcus anaerobius, Enterococcus faecalis as well as Helicobacter pylori. These bacteria have been described as colorectal cancer-associated pathogens $[6,10]$. Moreover, the low level of Bifidobacteria and high level of Clostridia in patients who experienced diarrhoea during EN were noted $[3,11]$. It should be emphasized that Clostridia is known as pathogenic bacteria causing diarrhoea [3].

Currently, the administration of probiotics is one of the methods used to modify gut microbiota [12, 13]. Lactobacillus plantarum 299v DSM 9843 (Lp299v) is a probiotic strain belonging to the Firmicutes and Gram-positive lactic acid bacteria $[14,15]$. It is commonly found in diet and is also able to reside in human colonic mucosa in vivo due to a specific mechanism of mannose adhesion [16]. Lp299v demonstrates high tolerance to acidic environments in gastric as well as alkaline in the duodenum [17]. It has immunomodulatory properties increasing the anti-inflammatory IL-10 synthesis and secretion. Moreover, it increases the transcription MUC2 and MUC3, thus secretion of mucins being glycoproteins, which provide protection for the intestinal mucosal surfaces [18]. Lp299v has antibacterial activity against pathogens, such as Listeria monocytogenes, Escherichia coli, Enterococcus faecalis as well as Clostridium difficile [17]. Kujawa-Szewieczek et al. trial has confirmed that routine use of Lp299v may prevent Clostridium difficile infection during antibiotic therapy in nephrology and transplantation ward [14]; these results were also confirmed in one-year extended study [19]. Lp299v is also recommended for patients suffering from irritable bowel syndrome (IBS). Its administration has been found to relieve symptoms, such as relapsing abdominal pain, flatulence, and diarrhoea [20].

As it was mentioned above, gastrointestinal symptoms occurring during EN are similar to those in IBS. The data regarding EN in combination with probiotics are limited; moreover, there is no data assessing the role of Lp299v in cancer patients receiving HEN. The primary aim of this randomized, double-blind, and placebo-controlled study was to determine the effect of the Lp299v on nutritional status of cancer 
patients receiving HEN. The secondary aims were to assess the role of Lp299v in the improvement of EN tolerance and patients' QOL.

\section{Methods}

\section{Patients}

Participants $(\mathrm{n}=35)$ were recruited by surgeon and nutritionist in Nutritional Counselling Centre Copernicus in Gdansk and Department of Clinical Nutrition and Dietetics (Medical University of Gdansk), Poland. Inclusion criteria were: age $\geq 18$ year., the presence of cancer, artificial access to the alimentary tract (naso-gastric tube, gastrostomy, percutaneous endoscopic gastrostomy, jejunostomy, microjejunostomy), qualification for HEN, written consent to take part in the study. Exclusion criteria included: age $<18$ year., patients requiring home parenteral nutrition, not being able to attend the visit in the study centre.

\section{Study design}

This study was designed as a randomized, double-blind, and placebo-controlled to assess the efficiency of Lp299v in cancer patients receiving HEN. Treatment duration was 4 weeks with 1 visit after completing the 4th week. The study protocol has been approved by the Independent Bioethics Committee for Scientific Research at the Medical University of Gdansk (identifier: 422/2016). All participants gave written informed consent prior to participation in this study and the information about this trial was explained to them. This study has been registered in ClinicalTrials.gov (identifier: NCT03940768).

The flow chart of this study is presented in Fig. 1. A total of 35 participants were randomized into two groups: one group receiving probiotic - Lp299v $(n=21)$ and the control group receiving placebo $(n=14)$. Among these 35 participants, 10 were excluded, because they did not complete the 4 weeks treatment due to resignation or death.

\section{Study products}

The study test product was Sanprobi IBS ${ }^{8}$ containing $10^{10}$ CFU of Lp299v (Sanprobi IBS $\circledast$ Sanprobi Sp. z o.o., Sp. k., Szczecin, Poland; producer of powder - Institute Rosell-Lallemand, Montreal, Canada; LP299v owner of probiotic strain - Probi AB, Lund, Sweden). Placebo was produced and packed by the same company and did not contain any microorganisms. Placebo capsule weighed $410 \mathrm{mg}+/-7.5 \%$ and contained potato starch - $403 \mathrm{mg}$ and magnesium stearate (magnesium salts of fatty acids) $-7 \mathrm{mg}$. Both, the test and placebo product had the same appearance, structure, and taste. The study products were stored at refrigerator temperature. The participants were instructed to take one capsule of probiotic/placebo product in the morning after breakfast and one capsule in the evening after dinner for 4 weeks. The patients who could not swallow were provided by mixed capsules' powder with $20 \mathrm{ml}$ of water 
or saline (in case of patients with jejunostomy and microjejunostomy). All participants were given standard normo-caloric enteral formulas with no additional fiber (average dose - $1500 \mathrm{ml}$ ) during 4-week therapy. The provision of enteral formula was adjusted to calorie requirements calculated with HarrisBenedict Equation. The advised speed of administration was:

a) jejunostomy $-30-40 \mathrm{ml} / \mathrm{h}$ during first $4-7$ days than reaching maximum tolerable dose/h (maximum $120 \mathrm{ml} / \mathrm{h}$ );

b) gastric access - repeated $-200-300 \mathrm{ml}$ bolus followed by a $2-3 \mathrm{~h}$ break.

\section{Sample size and randomization}

After meeting the inclusion criteria and obtaining consent agreement, participants were randomized to receive a probiotic or placebo product. The randomization ratio was 1:1 and was performed by means of researcherandomizer.com software, typically used by clinical research associates. The researches and participants were blinded to the treatment arm.

When computing a priori sample size, we anticipated that the probiotic intervention will decrease the weight loss by $10 \%$ with an SD of around $15 \%$ for weight change. Calculating the mean weight loss in a $70 \mathrm{~kg}$ men and assuming 1:1 allocation ratio and $80 \%$ statistical power we evaluated that the number of participants will be 36 . We randomly allocated 35 participants to receive either probiotic product or placebo. The required sample size was evaluated using the G-power analysis software.

\section{Outcomes}

\section{Primary outcomes}

The primary outcome was the improvement of nutritional status in a probiotic-receiving group in comparison to a placebo-receiving patients. The nutritional status was evaluated by means of anthropometric and laboratory parameters as well as the Nutritional Risk Screening 2002 tool (NRS 2002 tool). Anthropometric parameters were: the percentage of unintentional weight loss during last the 6 months before intervention, the composition of body mass (fat mass, muscle mass, total body water - TBW) and Body Mass Index (BMI). The body mass analysis was performed using BIA analyser - Medical Jawon in Department of 
Clinical Nutrition and Dietetics, Medical University of Gdansk, Poland. Laboratory tests included the serum concentration of albumin, total protein, and total lymphocyte count (TLC). The blood samples were taken in Nutritional Counselling Centre Copernicus in Gdansk and next given to the laboratory to conduct the analysis The anthropometric parameters and laboratory analyses were collected at baseline and after 4 weeks. The anthropometric parameters were noted at baseline and after 4 weeks.

Moreover, by means of NRS 2002 we evaluated nutritional status of study participants but only at baseline.

Malnutrition was diagnosed and categorized according to the Global Leadership Initiative on Malnutrition (GLIM) criteria (Stage 1 - moderate malnutrition, Stage 2 - severe malnutrition). The severity grading is based on phenotypic criteria. Stage 1 requires one of the following criteria: weight loss (5-10\% within the past 6 months, or $10-20 \%$ beyond 6 months), low BMI $\left(<20 \mathrm{~kg} / \mathrm{m}^{2}\right.$ if $<70$ year., $<$ $22 \mathrm{~kg} / \mathrm{m}^{2}$ if $\geq 70$ year.), and reduced muscle mass (mild to moderate deficit). Stage 2 - one of the following phenotypic criterion must be met: weight loss (>10\% within the past 6 months or $>20 \%$ beyond 6 months), low BMI (< $18.5 \mathrm{~kg} / \mathrm{m}^{2}$ if $<70$ year., $<20 \mathrm{~kg} / \mathrm{m}^{2}$ if $\geq 70$ year.), and reduced muscle mass (severe deficit).

\section{Secondary outcomes}

The secondary outcomes were the improvement of EN tolerance and patients' quality of life. The tolerance of EN was assessed with authors own questionnaire referring to number of stools, frequency of vomiting and flatulence. The questionnaire was filled every day by patients and analysed during the follow-up visit after 4 weeks. In order to assess patients' quality of life, we used the World Health 
Organization Quality of Life-BREF (WHOQOL-BREF) questionnaire. It contains 26 questions divided into 4 domains (D1 - environmental, D2 - psychological, D3 - somatic, and D4 - social factors); moreover, first question (Q1 - How would you rate your quality of life?") regards patients' self-assessment of QOL (where 1 points "very poor" and 5 "very good") and second (Q2 - "How satisfied are you with your health?") patients' self-assessment of status of health (where 1 point means "very dissatisfied" and 5 "very satisfied"). The QOL was checked at baseline and after 4 weeks. The more points, the better QOL.

\section{Statistical analysis}

The statistical analyses have been performed using the statistical suite StatSoft Inc. 2014 STATISTICA version 12.0. www.statsoft.com and Microsoft Excel. The quantitative variables were characterized by the arithmetic mean of standard deviation or median or max/min (range) and $95 \%$ confidence interval. The qualitative variables were presented with the use of count and percentage. In order to check if a quantitative variable derives from a population of normal distribution the W Shapiro-Wilk test has been used. Whereas to prove the hypotheses on homogeneity of variances Leven (Brown-Forsythe) test has been utilized. Statistical significance of differences between two groups (unpaired variables model) was processed with the t-Student test (or Welch test in the case of lack of homogeneity) or U Mann-Whitney test (in cases where conditions of performing the t-Student test were not satisfied or for variables measured by ordinal scale). The significance of difference between more than two groups were assessed with $\mathrm{F}$ test (ANOVA) or Kruskal-Wallis (if AVOVA conditions were not fulfilled). In the case of statistically significant differences between two groups post hoc tests were utilized (Tukey test for $F$ or Dunn for Kruskal-Wallis). In the case of two paired variables model t-Student or Wilcoxon signed-rank (if t-Student conditions are not fulfilled or for variables measured in ordinal scale) test was utilized. The significance of difference between more than two variables in the paired variables model has been checked by analysis of variance with repeated measurements or by Friedman test (if analysis of variance conditions are not satisfied or for variables measured in ordinal scale). Chi-squared tests for independence were used for qualitative variables (with the use of Yates correction for cell counts below 10, with check of Cochrane's conditions or with Fisher's exact test respectively). In order to determine dependence, strength and direction between variables, correlation analysis was used by determining the Pearson or Spearman's correlation coefficients. In all the calculations the statistical significance level of $p=0.05$ has been used.

\section{Results}

\section{Patients' baseline characteristics}

A total of 35 participants were included in this trial (Table 1). Among these, 10 were excluded due to resignation or death. $82.85 \%$ of ITT patients and $80 \%$ of FAS were severe malnourished according to GLIM criteria before intervention. No significant differences were observed between the Lp299v groups and the placebo group in terms of age, gender, type of cancer, artificial access to the alimentary tract, NRS 2002 , and percent of unintentional weight loss during last 6 months at baseline. 
Table 1

Patients' characteristics ITT - Intention to treat; FAS - Full set analysis; PEG - percutaneous endoscopic gastrostomy; NRS 2002 - Nutritional Risk Screening; BMI - Body Mass Index.

\begin{tabular}{|c|c|c|c|c|c|c|}
\hline & ITT & FAS & ITT & FAS & ITT & FAS \\
\hline & \multicolumn{2}{|l|}{ Lp299v } & \multicolumn{2}{|l|}{ Placebo } & \multirow[t]{2}{*}{$P$-value } & \\
\hline & $\mathrm{n}=21$ & $\mathrm{n}=15$ & $\mathrm{n}=14$ & $n=10$ & & \\
\hline Age (yr.) & $60 \pm 10.9$ & $\begin{array}{l}60.9 \pm \\
12.2\end{array}$ & $\begin{array}{l}61.1 \pm \\
8.9\end{array}$ & $\begin{array}{l}62.2 \pm \\
10.2\end{array}$ & 0.7566 & 0.7887 \\
\hline Gender (M/F) & $16 / 5$ & $10 / 5$ & $11 / 3$ & $8 / 2$ & 0.8695 & 0.4670 \\
\hline $\begin{array}{l}\text { Disease (\%) } \\
\text { Gastric cancer } \\
\text { Esophageal cancer } \\
\text { Throat cancer } \\
\text { Tongue cancer } \\
\text { Tonsil cancer } \\
\text { Gum cancer } \\
\text { Sinus cancer } \\
\text { Lung cancer } \\
\text { Pancreatic cancer } \\
\text { Craniofacial cancer }\end{array}$ & $\begin{array}{l}19 \\
38.1 \\
19 \\
4.8 \\
9.5 \\
4.8 \\
4.8 \\
0 \\
0 \\
0\end{array}$ & $\begin{array}{l}33.3 \\
20 \\
20 \\
6.7 \\
0 \\
6.7 \\
6.7 \\
6.7 \\
0 \\
0\end{array}$ & $\begin{array}{l}21.4 \\
21.4 \\
28.6 \\
14.3 \\
0 \\
0 \\
0 \\
7.1 \\
7.1 \\
7.1\end{array}$ & $\begin{array}{l}30 \\
20 \\
10 \\
20 \\
0 \\
0 \\
0 \\
10 \\
10 \\
0\end{array}$ & 0.5992 & 0.6830 \\
\hline $\begin{array}{l}(\%) \\
\text { PEG } \\
\text { Jejunostomy } \\
\text { Microjejunostomy } \\
\text { Naso-gastric tube }\end{array}$ & $\begin{array}{l}47.6 \\
47.6 \\
0 \\
4.8\end{array}$ & $\begin{array}{l}53.3 \\
46.7 \\
0 \\
0\end{array}$ & $\begin{array}{l}42.9 \\
28.6 \\
7.1 \\
21.4\end{array}$ & $\begin{array}{l}40 \\
30 \\
10 \\
20\end{array}$ & 0.2265 & 0.1619 \\
\hline $\begin{array}{l}(\%) \\
\text { NRS } 2002 \text { tool score } \\
<3 \\
3 \\
4 \\
5 \\
6\end{array}$ & $\begin{array}{l}0 \\
4.8 \\
19 \\
61.9 \\
14.3\end{array}$ & $\begin{array}{l}0 \\
6.7 \\
20 \\
53.3 \\
20\end{array}$ & $\begin{array}{l}0 \\
7.1 \\
21.4 \\
50 \\
21.4\end{array}$ & $\begin{array}{l}0 \\
0 \\
30 \\
50 \\
20\end{array}$ & 0.9043 & 0.8183 \\
\hline $\mathrm{BMI}\left(\mathrm{kg} / \mathrm{m}^{2}\right)$ & $\begin{array}{l}21.2 \pm \\
3.4\end{array}$ & $22 \pm 3.1$ & $\begin{array}{l}21.4 \pm \\
3.9\end{array}$ & $\begin{array}{l}21.5 \pm \\
3.6\end{array}$ & 0.8916 & 0.7083 \\
\hline $\begin{array}{l}\% \text { of unintentional weight } \\
\text { loss (last } 6 \text { months) }\end{array}$ & $\begin{array}{l}19.3 \pm \\
9.9\end{array}$ & $\begin{array}{l}17.1 \pm \\
8.6\end{array}$ & $\begin{array}{l}14.7 \pm \\
6.4\end{array}$ & $\begin{array}{l}13.9 \pm \\
7.0\end{array}$ & 0.1305 & 0.3356 \\
\hline Albumin $(\mathrm{g} / \mathrm{L})$ & $\begin{array}{l}40.7 \pm \\
4.2\end{array}$ & $41.7 \pm 3$ & $\begin{array}{l}39.7 \pm \\
5.4\end{array}$ & $\begin{array}{l}39.7 \pm \\
6.2\end{array}$ & 0.5516 & 0.3571 \\
\hline Total protein $(\mathrm{g} / \mathrm{L})$ & $\begin{array}{l}69.9 \pm \\
4.2\end{array}$ & $\begin{array}{l}70.6 \pm \\
3.7\end{array}$ & $\begin{array}{l}68.6 \pm \\
5.0\end{array}$ & $69.1 \pm 4$ & 0.4293 & 0.3362 \\
\hline TLC/ L & $\begin{array}{l}1620.2 \pm \\
898.2\end{array}$ & $\begin{array}{l}1628.5 \pm \\
809.9\end{array}$ & $\begin{array}{l}1286.7 \pm \\
666.9\end{array}$ & $\begin{array}{l}1454.4 \pm \\
411.2\end{array}$ & 0.2440 & 0.5379 \\
\hline
\end{tabular}

Nutritional status 


\section{Laboratory parameters}

The mean changes within the laboratory parameters over 4 week period in both groups are shown in Fig. 2. The increase of the level of albumin in blood serum was significantly higher in Lp299v group than in the placebo group after week 4 (baseline $41.7 \pm 3$ vs $39.7 \pm 6.2$; after treatment $43.2 \pm 4.3$ vs $38 \pm 7.1$; $p$ $=0.032$; respectively). In Lp299v group the concentration of albumin in blood serum was significantly increased after week 4 in compared to baseline $(p=0.0335)$; but not in placebo group $(p=0.594)$. Overall increase of total protein after 4 week in comparison with baseline was also observed in Lp299v group, however it was not statistically significant $(70.6 \pm 3.7$ vs $71.4 \pm 4.7 ; p>0.05)$. This improvement was not present in placebo group $(69.1 \pm 4$ vs $67.6 \pm 8.8 ; p>0.05)$. No statistically significant differences were found after treatment period in TLC/L in Lp299v and placebo group (1628.5 \pm 809.9 vs $1454.4 \pm 411.2$; $1508.6 \pm 938.3$ vs $1278.5 \pm 640.1 ; p>0.05$; respectively).

\section{Anthropometric parameters}

The alterations of anthropometric parameters are presented in Table 3. There were no statistically significant changes in body mass, BMI, the content of fat mass, muscle mass, and TBW in both groups after 4 week treatment $(p>0.05)$.

Table 3

Changes in anthropometric parameters in both groups. TBW - total body water, BMI - Body Mass Index

\begin{tabular}{|c|c|c|c|c|c|c|}
\hline & Lp299v & & Placebo & & $P$-value & \\
\hline $\begin{array}{l}\text { Anthropometric } \\
\text { parameters }\end{array}$ & Baseline & $\begin{array}{l}\text { After } 4 \\
\text { weeks }\end{array}$ & Baseline & $\begin{array}{l}\text { After } 4 \\
\text { weeks }\end{array}$ & Baseline & $\begin{array}{l}\text { After } 4 \\
\text { weeks }\end{array}$ \\
\hline Body mass $(\mathrm{kg})$ & $\begin{array}{l}65.7 \pm \\
12.1\end{array}$ & $65 \pm 10.8$ & $\begin{array}{l}64.5 \pm \\
11.1\end{array}$ & $63.5 \pm 11.8$ & 0.8056 & 0.7461 \\
\hline $\mathrm{BMI}\left(\mathrm{kg} / \mathrm{m}^{2}\right)$ & $\begin{array}{l}22.0 \pm \\
3.1\end{array}$ & $21.7 \pm 3.0$ & $\begin{array}{l}21.5 \pm \\
3.6\end{array}$ & $21.1 \pm 3.7$ & 0.7083 & 0.6775 \\
\hline Fat mass (\%) & $\begin{array}{l}14.2 \pm \\
6.3\end{array}$ & $13.8 \pm 6.3$ & $\begin{array}{l}13.6 \pm \\
6.8\end{array}$ & $13.3 \pm 6.6$ & 0.8094 & 0.8352 \\
\hline Muscle mass $(\mathrm{kg})$ & $\begin{array}{l}51.3 \pm \\
8.7\end{array}$ & $50.6 \pm 7.9$ & $\begin{array}{l}50.4 \pm \\
7.6\end{array}$ & $49.7 \pm 7.2$ & 0.2673 & 0.2809 \\
\hline TBW (\%) & $\begin{array}{l}36.8 \pm \\
6.3\end{array}$ & $36.4 \pm 5.7$ & $\begin{array}{l}36.3 \pm \\
5.3\end{array}$ & $35.8 \pm 4.9$ & 0.8274 & 0.8019 \\
\hline
\end{tabular}

\section{Tolerance of enteral nutrition}

The frequencies of vomiting and flatulence (Fig. 3) were reduced with statistical difference at week 4 compared to baseline in Lp299v group (baseline: $1.5 \pm 2$, after 4 week: $0.6 \pm 1.8, p=0.0346$; baseline: 1.8 
\pm 2 .2, after 4 week: $0.5 \pm 1, p=0.0117$; vomiting and flatulence respectively); but not in placebo group (baseline: $1.9 \pm 1.9$, after 4 week: $1.1 \pm 2.2, p=0.1415$; baseline: $1 \pm 1.5$, after 4 week: $0.1 \pm 0.3, p=0.0679$, vomiting and flatulence respectively). The significant differences in frequency of vomiting and flatulence at baseline, at week 2,3 , and 4 between both groups were not observed $(p=0.5235, p=0.1741, p=$ $0.5603, p=0.4054 ; p=0.4540, p=0.8244, p=0.6373, p=0.4540$, respectively).

The alterations of the stools frequency (Fig. 3) per day were significantly reduced at week 4 compared to baseline in both groups (Lp299v baseline: $1.3 \pm 0.7$, after 4 week: $0.8 \pm 0.4, p=0.029$; placebo baseline: 1 \pm 0.4 , after 4 week: $0.8 \pm 0.4, p=0.0295$ ). No differences in changes of stools frequency at baseline, week 2,3 , and 4 between both groups were noted ( $p=0.1548, p=0.3892, p=0.33, p=0.8852$, respectively).

\section{Quality of life}

The alterations of quality of life are shown in Fig. 4. The quality of life in Q1 was significantly increased in Lp299v group after week 4 compared to baseline $(2.7 \pm 1$ vs $3.3 \pm 0.8, p=0.0077)$. In Lp299v group the QOL was significantly higher in D2 and D4 after week 4 compared to baseline $(12.7 \pm 2$ vs $13.5 \pm 2, \mathrm{p}=$ $0.028 ; 14.4 \pm 2.1$ vs $15.4 \pm 1.9, \mathrm{p}=0.0414$; respectively). In placebo group the increase of OQL was also observed in Q1, Q2, D2, D3, and D4, however, the differences were not significant compared to baseline. Moreover, no statistically significant differences between both groups in Q1, Q2, and domains were noted $(p>0.05)$.

\section{Discussion}

The previous studies have reported that adding probiotics to EN could improve immune function and decrease the incidence of diarrhoea in cancer patients [3, 21]. Yi et al. conducted a meta-analysis which confirmed that early EN supplementation with probiotics effectively decreased the risk of infections (risk ratio [RR], 0.53; 95\% confidence interval [Cl], 0.44-0.65), mortality ( $R R, 0.56 ; 95 \% \mathrm{Cl}, 0.38-0.82$ ), gastrointestinal complications (RR, $0.19 ; 95 \% \mathrm{Cl}, 0.13-0.25)$, and shortened an intensive care unit stay (mean difference [MD], $-4.55 ; 96 \% \mathrm{Cl},-5.91$ to - 3.19) in patients with severe head injury including also cancer patients [22]. Notwithstanding, the effect of the Lp299v on nutritional status and the improvement of EN tolerance as well as the quality of life of cancer patients receiving HEN has not been studied earlier. Moreover, the present study treatment length is 4 weeks in comparison with other studies with shortened observation period. At the beginning, the administration of probiotic was planned for 12 weeks as it was previously registered in ClinicalTrials.gov before intervention; however, we decided to reduce the treatment period to 4 weeks, due to high mortality of cancer patients qualified for HEN.

Probiotics can reduce gastrointestinal symptoms, such as nausea, bloating, and diarrhoea [20, 23]. In Zhao et al. prospective randomized and controlled trial, it was shown that a combination of fiber and probiotics with EN significantly reduced the incidence of diarrhoea associated with EN in postoperative patients with gastric cancer [3]. The incidence of diarrhoea during 7 days treatment was $60 \%$ in patients of FF group (fiber-free nutrition formula), $30 \%$ of FE (fiber-enriched nutrition formula), and $5 \%$ of FEP 
(fiber-and probiotic-enriched nutrition formula). The similar results were obtained by Xie et al. confirming that diarrhoea caused by EN occurred less frequently in the gastric cancer patients receiving probiotics compared to controls [21]. Importantly, studies have shown that probiotics efficacy is strain-specific [24]. Lönnermark et al. in a double-blind, placebo-controlled trial investigated the effect of Lp299v (in a dose $10^{10} \mathrm{CFU}$ per day) on the incidence of antibiotic-associated gastrointestinal symptoms [25]. The significant reduction of the frequency of loose stools (odds ratio (OR), 0.69; 95\% confidence interval (Cl), $0.52-0.92 ; \mathrm{P}=0.012)$ and nausea $(\mathrm{OR} 0.51 ; 95 \% \mathrm{Cl}, 0.30-0.85 ; \mathrm{P}=0.0097)$ after administration of Lp299v was noted [25]. In the current study, the frequency of vomiting, flatulence, and stools were significantly reduced at week 4 compared to baseline in Lp299v group $(p=0.0346, p=0.0117, p=0.024$, respectively); however, the significant differences between Lp299v and placebo group were not observed. Moreover, the frequency of flatulence was reduced week-by-week in Lp299v group and the number of stools started to decline in week 3 and 4 , which was not noted in the placebo group. It may be associated with the modification of gut microbiota after administration of Lp299v. To sum up, the administration of Lp299v potentially may reduce the gastrointestinal symptoms in patients receiving HEN.

According to the European Society for Clinical Nutrition and Metabolism (ESPEN) guidelines, all cancer patients should be screened regularly for the risk of malnutrition [26]. In the above mentioned study by Zhao et al., the assessment of nutritional status in patients with gastric cancer $(n=120)$ receiving EN for 7 days was based on BMI (FF group $21.41 \pm 2.20 \mathrm{~kg} / \mathrm{m}^{2}$, FE 21.73 \pm 2.65 , FEP $21.83 \pm 3.12 ; \mathrm{p}=0.89$ ) and laboratory parameters (the level of albumin - FF $37.71 \pm 2.72 \mathrm{~g} / \mathrm{L}, \mathrm{FE} 37.01 \pm 2.73$, FEP $36.30 \pm 3.28, \mathrm{p}=$ 0.34; prealbumin - FF $192 \pm 6.72 \mathrm{mg} / \mathrm{L}$ FE $188 \pm 8.41$, FEP $188 \pm 7.43, \mathrm{p}=0.16$; transferrin - FF $1.83 \pm$ $0.27 \mathrm{mg} / \mathrm{L}, \mathrm{FE} 1.70 \pm 0.31$, FEP $1.85 \pm 0.35, \mathrm{p}=0.27$; total lymphocyte count $-\mathrm{FF} 1.25 \pm 0.42 \times 10^{9} / \mathrm{L}$, FE $1.15 \pm 0.34$, FEP $1.13 \pm 0.32, p=0.53$ ) [3]. The statistically significant difference between 3 groups in terms of these laboratory parameters after 7 days of EN was not observed [3]. Similarly, in Xie et al. study including patients with gastric cancer $(n=140)$ receiving EN in combination with probiotics or placebo for 8 days, no difference was found between two groups after the treatment period [21]. In the current study, it was confirmed that Lp299v significantly increased the level of albumin in the blood serum in comparison with the placebo group $(p=0.032)$; however, the significant increase of the level of total protein was not observed $(p>0.05)$. Similarly, no significant changes were observed in TLC. The half-life of albumin is around 21 days. Therefore, it is not accurate as a laboratory parameter to determine short term alterations. This may be the reason why in the current study the changes of this parameter were observed on the contrary to the above mentioned studies (4 weeks vs 7 and 8 days). The concentration of albumin - being a negative acute phase protein - is decreased not only in malnutrition but also in the presence of inflammation [27]. The increase of the level of albumin after administration of Lp299v can suggest improvement in the nutritional status and/or the reduction of inflammation. Due to nonsignificant improvement in anthropometric measures, increase in albumin concentration during Lp299v can be interpreted as anti-inflammatory effect of probiotic administration. However, other laboratory parameters describing the inflammation, such as C-reactive protein or pro- and anti-inflammatory cytokines were not included in this study, thus the immunomodulatory effect of Lp299v in cancer patients 
needs further studies. The lack of significant improvement in BMI, fat mass, muscle mass, and TBW after 4 week administration of Lp299v were observed.

GLIM initiative proposed severity grading of malnutrition into moderate and severe stages [28]. It has been published in 2019; therefore, data regarding clinical complications of those criteria and cancer are limited. In 2019, Contreras-Bolivar et al. presented results of observational and prospective study, which confirmed that SGA (Subjective Global Assessment) and GLIM criteria (mainly with hand grip strength) are useful in diagnosing malnutrition having also similar predictive value regarding six-month mortality in cancer inpatients [29]. In the current study, $82.85 \%$ of ITT patients and $80 \%$ of FAS were severely malnourished according to GLIM criteria before intervention; therefore, it was almost impossible to improve their nutritional status.

The improvement of patients' quality of life is one of the most important goals of multi-disciplinary anticancer therapy. The effects of 3 months HEN on QOL and nutritional status after esophagectomy were assessed by Wu et al. [30]. The participants were divided into 2 groups: undergoing Ivor Lewis minimally invasive esophagectomy with laparoscopic jejunal feeding tube placement (MIE group) and patients after open esophagectomy with naso-jejunal feeding tube placement (OE group). The results of this study confirmed that patients who received HEN had a lower risk of malnutrition compared to patients who did not receive HEN (PG-SGA score, 5.7 vs 7.9, $\mathrm{p}<0.01$ ). The QLQ-30 questionnaire including physical, emotional, and cognitive functioning was used to evaluate the QOL. The mean scores of the global quality of life, physical function, role function, and social function were significantly higher in the MIE group compared to the OE group. It was concluded that after 3 months of HEN, patients in the MIE group had fewer symptoms and superior improvements in functioning in comparison to patients of the $O E$ group [30]. Moreover, probiotics have been tested to improve cancer patients' quality of life. In doubleblind, randomized, and placebo-controlled trial it was noted that administration of probiotics per 12 weeks improve cancer-related quality of life - Functional Assessment of Cancer Therapy (FACT) (baseline vs. 12 weeks: $19.79 \pm 4.66$ vs. $21.18 \pm 3.67, p=0.04$ ) and fatigue-related FACT (baseline vs. 12 weeks: $43.00(36.50-45.50)$ vs. $44.50(38.50-49.00), p=0.02)$ in colorectal cancer survivors [31]. Ohigashi et al. study also confirmed that the administration of probiotics (containing Bacillus natto and Lactobacillus acidophilus) for 3 months may be effective in the improvement of QOL after colorectal resection $(n=77)$ [32]. However, none of the previous studies tested the efficacy of Lp299v in the cancer patient population. It was highly desirable since this strain was shown to be efficient in the reduction of gastrointestinal symptoms and as a consequence the improvement of QOL [33,34]. In the current study, the self-assessment of QOL was significantly increased after week 4 compared to baseline $(p=0.0077)$ in patients receiving Lp299v; moreover, the significant improvement in the psychological domain and social factors was also noted $(p=0.028 ; p=0.0414$, respectively). In the placebo group, the improvement of $Q O L$ in Q1, Q2, psychological, somatic, and social factors were also noted; however, the differences were not significant compared to baseline. Furthermore, statistically significant differences between both groups were not observed ( $p>0.05)$; therefore, it is not certain if the improvement of QOL in Lp299v is associated with the administration of probiotic or it is the results of EN. This study only indicated that Lp299v was not inferior to placebo in changing QOL in cancer patients receiving HEN. 
The present study has some limitations. The most important one is that this trial was conducted in a single centre with small sample size; moreover, the group was non-homogenous. We planned to recruit 40 participants as it was previously declared in ClinicalTrials.gov. However, eventually we recruited 35 patients and completed the current study in ClinicalTrials.gov regarding this number of participants. It is difficult and takes a lot of time to include a larger number of participants especially with the same type of cancer for 4 week treatment period, among others due to receive consent agreement and high mortality of cancer patients qualified for HEN. Therefore, there is a need to create a multi-centre trial with larger sample size. A noticeable fact is that the majority of participants from the study were patients with advanced incurable cancer on palliative treatment. Improvement of nutritional status in this group is generally difficult or impossible to achieve. The main goal of nutritional treatment in this stage of the disease is to positively influence the quality of life.

\section{Direction for future studies}

This study showed possible directions for future investigations. Targeting a specific homogenous group of patients may bring more conclusive results. In our opinion individuals with early stages of cancer and good nutritional status/mild malnutrition may benefit more from probiotic intervention. Identification of microbiota changes will possibly enable researchers to determine the desired intervention method and study group.

\section{Conclusions}

In summary, in the current study we have demonstrated that administration of Lp299v in cancer patients receiving HEN may improve laboratory parameters mainly the concentration of albumin, however overall it does not have an impact on nutritional status. Lp299v may reduce the gastrointestinal symptoms related to EN; notwithstanding, the improvement of QOL may be the result of EN rather than the effect of administration of Lp299v.

\section{Declarations}

Ethics approval and consent to participate

This study has been approved by Independent Bioethics Committee for Scientific Research at Medical University of Gdańsk, Poland (the project indentification code: 422/2016). The participants was informed about this study, potential benefits and risks. The consent agreement received from patients was necessary to include them to the trial.

Consent for publication

Not applicable.

Availability of data

The data confirming the findings of this study are available in Department of Surgical Oncology, Medical University of Gdańsk, Mariana Smoluchowskiego 17, 80-214 Gdańsk. The data are available from author 
K.K.-S.

Competing interest

K.S.-Ż. receives renumeration from probiotic company.

Funding

The study products (probiotic and placebo) were provided by International Pharmaceutical Consulting Sp.

z o.o., Sp. k. Szczecin, Poland. This company also covered the cost of publication.

Author's contribution

Conceptualization, K.K.-S. and M.F.; Data curation, K.K.-S., M.F., J.R., K.K.Ż, W.S., and W.M.; Formal analysis, K.K.-S, J.R.; Investigation, K.K.-S., M.F., and W.S.; Methodology, K.K.-S.; Resources, K.K.-S., M.F., and J.R.; Supervision, M.F., K.S.-Ż., and W.M.; Writing - original draft, K.K.-S.; Writing - review \& editing, M.F., K.S.-Ż., J.R., W.S., and W.M.

Acknowledgment

The authors thank International Pharmaceutical Consulting Sp. z o.o., Sp. k. Szczecin, Poland for supplying the Lactobacillus plantarum $299 \mathrm{v}$ capsules and placebo.

\section{Abbreviations}

QOL: quality of life

HEN: home enteral nutrition

EN: enteral nutrition

Lp299v : Lactobacillus plantarum 299v (DSM 9843)

ITT: intention to treat

FAS: full set analysis

NRS 2002: Nutritional Risk Screening 2002

TBW: total body water

BMI: body mass index

TLC total lymphocyte count

GLIM: Global Leadership Initiative on Malnutrition

WHOQOL-BREF: World Health Organization Quality of Life-Bref

PEG: percutaneous endoscopic gastrostomy 
ESPEN: European Society for Clinical Nutrition and Metabolism

FF: fiber-free nutrition formula

FE: fiber-enriched nutrition formula

FEP: fiber- and probiotic-enriched nutrition formula

Cl: confidence interval

SGA: Subjective Global Assessment

MIE: Ivor Lewis minimally invasive esophagectomy

OE: open esophagectomy

FACT: Functional Assessment of Cancer Therapy

\section{References}

1. Gavazzi C, Colatruglio S, Valoriani F, Mazzaferro V, Sabbatini A, Biffi R, et al. Impact of home enteral nutrition in malnourished patients with upper gastrointestinal cancer: A multicentre randomised clinical trial. Eur J Cancer Oxf Engl. 2016;64:107-12.

2. Villar Taibo R, Martínez Olmos M, Bellido Guerrero D, Vidal Casariego A, Peinó García R, Martís Sueiro A, et al. Epidemiology of home enteral nutrition: an approximation to reality. Nutr. Hosp. 2018; 3, 511-518.

3. Zhao R, Wang Y, Huang Y, Cui Y, Xia L, Rao Z, et al. Effects of fiber and probiotics on diarrhea associated with enteral nutrition in gastric cancer patients. Medicine. 2017;43:8418.

4. Wiesen P, Van Gossum A, Preiser JC. Diarrhoea in the critically ill. Curr Opin Crit Care. 2006;2:149-54.

5. McNaught CE, Woodcock NP, Anderson ADG, MacFie J. A prospective randomised trial of probiotics in critically ill patients. Clin Nutr Edinb Scotl. 2005;2:211-9.

6. Dai Z, Zhang J, Wu Q, Chen J, Liu J, Wang L, et al. The role of microbiota in the development of colorectal cancer. Int J Cancer. 2019;8:2032-41.

7. Garrett WS. Cancer and the microbiota. Science. 2015;6230:80-6.

8. Kaźmierczak-Siedlecka K, Fic M, Folwarski M, Makarewicz W. The role of human gut microbiota in carcinogenesis of gastrointestinal cancers. Diet \& Microbes: Gut health for the brain and body, 6th Nutrition Winter School 2020, Levi, Finland, 27-31 January 2020.

9. Kaźmierczak-Siedlecka K, Daca A, Fic M, Van de Wetering T, Folwarski M, Makarewicz W. Therapeutic methods of gut microbiota modification in colorectal cancer management - fecal microbiota transplantation, prebiotics, probiotics, and synbiotics. Gut Microbes 2020 (under review). 
10. Kaźmierczak-Siedlecka K, Ruszkowski J, Skonieczna-Żydecka K, Jędrzejczak J, Folwarski M, Makarewicz W. Gastrointestinal cancers: the role of microbiota in carcinogenesis and the role of probiotics and microbiota in the anti-cancer therapy efficiency. Cent Eur J Immunol. 2020 (accepted).

11. Whelan K, Judd PA, Preedy VR, Simmering R, Jann A, Taylor MA. Fructooligosaccharides and fiber partially prevent the alterations in fecal microbiota and short-chain fatty acid concentrations caused by standard enteral formula in healthy humans. J Nutr. 2005;8:1896-902.

12. Raman M, Ambalam P, Kondepudi KK, Pithva S, Kothari C, Patel AT, et al. Potential of probiotics, prebiotics and synbiotics for management of colorectal cancer. Gut Microbes. 2013;3:181-92.

13. Chen D, Wu J, Jin D, Wang B, Cao H. Fecal microbiota transplantation in cancer management: Current status and perspectives. Int J Cancer. 2019;8:2021-31.

14. Kujawa-Szewieczek A, Adamczak M, Kwiecień K, Dudzicz S, Gazda M, Więcek A. The Effect of Lactobacillus plantarum 299v on the Incidence of Clostridium difficile Infection in High Risk Patients Treated with Antibiotics. Nutrients. 2015;12:10179-88.

15. Kaźmierczak-Siedlecka K, Daca A, Folwarski M, Witkowski J, Bryl E, Makarewicz W. The role of Lactobacillus plantarum 299v in supporting treatment of selected diseases. Cent Eur J Immunol. 2020 (accepted).

16. Johansson ML, Molin G, Jeppsson B, Nobaek S, Ahrné S, Bengmark S. Administration of different Lactobacillus strains in fermented oatmeal soup: in vivo colonization of human intestinal mucosa and effect on the indigenous flora. Appl Environ Microbiol. 1993;1:15-20.

17. Goossens D, Jonkers D, Russel M, Thijs A, van den Bogaard A, Stobberingh E, et al. Survival of the probiotic, L. plantarum $299 \mathrm{v}$ and its effects on the faecal bacterial flora, with and without gastric acid inhibition. Dig Liver Dis. 2005;1:44-50.

18. Mack DR, Ahrne S, Hyde L, Wei S, Hollingsworth MA. Extracellular MUC3 mucin secretion follows adherence of Lactobacillus strains to intestinal epithelial cells in vitro. Gut. 2003;6:827-33.

19. Dudzicz S, Kujawa-Szewieczek A, Kwiecień K, Więcek A, Adamczak M. Lactobacillus plantarum 299v Reduces the Incidence of Clostridium difficile Infection in Nephrology and Transplantation WardResults of One Year Extended Study. Nutrients. 2018;11:1574.

20. Ducrotté P, Sawant P, Jayanthi V. Clinical trial: Lactobacillus plantarum 299v (DSM 9843) improves symptoms of irritable bowel syndrome. World J Gastroenterol. 2012;30:4012-8.

21. Xie H, Lu Q, Wang H, Zhu X, Guan Z. Effects of probiotics combined with enteral nutrition on immune function and inflammatory response in postoperative patients with gastric cancer. J BUON. 2018;3:678-83.

22. Yi LJ, Tian X, Shi B, Pi YP, Chen WQ. Early enteral nutrition supplemented with probiotics improved the clinical outcomes in severe head injury. Medicine. 2019;17:15426.

23. Wei $D$, Heus P, van de Wetering FT, van Tienhoven G, Verleye L, Scholten RJ. Probiotics for the prevention or treatment of chemotherapy- or radiotherapy-related diarrhoea in people with cancer. Cochrane Database Syst Rev. 2018;8:008831. 
24. McFarland LV, Evans CT, Goldstein EJC. Strain-Specificity and Disease-Specificity of Probiotic Efficacy: A Systematic Review and Meta-Analysis. Front Med. 2018; 5.

25. Lönnermark E, Friman V, Lappas G, Sandberg T, Berggren A, Adlerberth I. Intake of Lactobacillus plantarum reduces certain gastrointestinal symptoms during treatment with antibiotics. J Clin Gastroenterol. 2010;2:106-12.

26. Arends J, Bachmann P, Baracos V, Barthelemy N, Bertz $H$, Bozzetti F, et al. ESPEN guidelines on nutrition in cancer patients. Clin Nutr. 2017;1:11-48.

27. Gulhar R, Jialal I. Physiology, Acute Phase Reactants. Stat Pearls. 2019.

28. Cederholm T, Jensen GL, Correia MITD, Gonzalez MC, Fukushima R, Higashiguchi T, et al. GLIM criteria for the diagnosis of malnutrition - A consensus report from the global clinical nutrition community. Clin Nutr. 2019;1:1-9.

29. Contreras-Bolívar V, Sánchez-Torralvo FJ, Ruiz-Vico M, González-Almendros I, Barrios M, Padín S, et al. GLIM Criteria Using Hand Grip Strength Adequately Predict Six-Month Mortality in Cancer Inpatients. Nutrients. 2019;9:2043.

30. Wu Z, Wu M, Wang Q, Zhan T, Wang L, Pan S, et al. Home enteral nutrition after minimally invasive esophagectomy can improve quality of life and reduce the risk of malnutrition. Asia Pac J Clin Nutr. 2018;1:129-36.

31. Lee JY, Chu SH, Jeon JY, Lee MK, Park JH, Lee DC, et al. Effects of 12 weeks of probiotic supplementation on quality of life in colorectal cancer survivors: a double-blind, randomized, placebo-controlled trial. Dig Liver Dis. 2014;12:1126-32.

32. Ohigashi S, Hoshino Y, Ohde S, Onodera H. Functional outcome, quality of life, and efficacy of probiotics in postoperative patients with colorectal cancer. Surg Today. 2011;9:1200-6.

33. Nobaek S, Johansson ML, Molin G, Ahrné S, Jeppsson B. Alteration of intestinal microflora is associated with reduction in abdominal bloating and pain in patients with irritable bowel syndrome. Am J Gastroenterol. 2000;5:1231-8.

34. Niedzielin K, Kordecki H, Birkenfeld B. A controlled, double-blind, randomized study on the efficacy of Lactobacillus plantarum 299V in patients with irritable bowel syndrome. Eur J Gastroenterol Hepatol. 2001;10:1143-7.

\section{Figures}




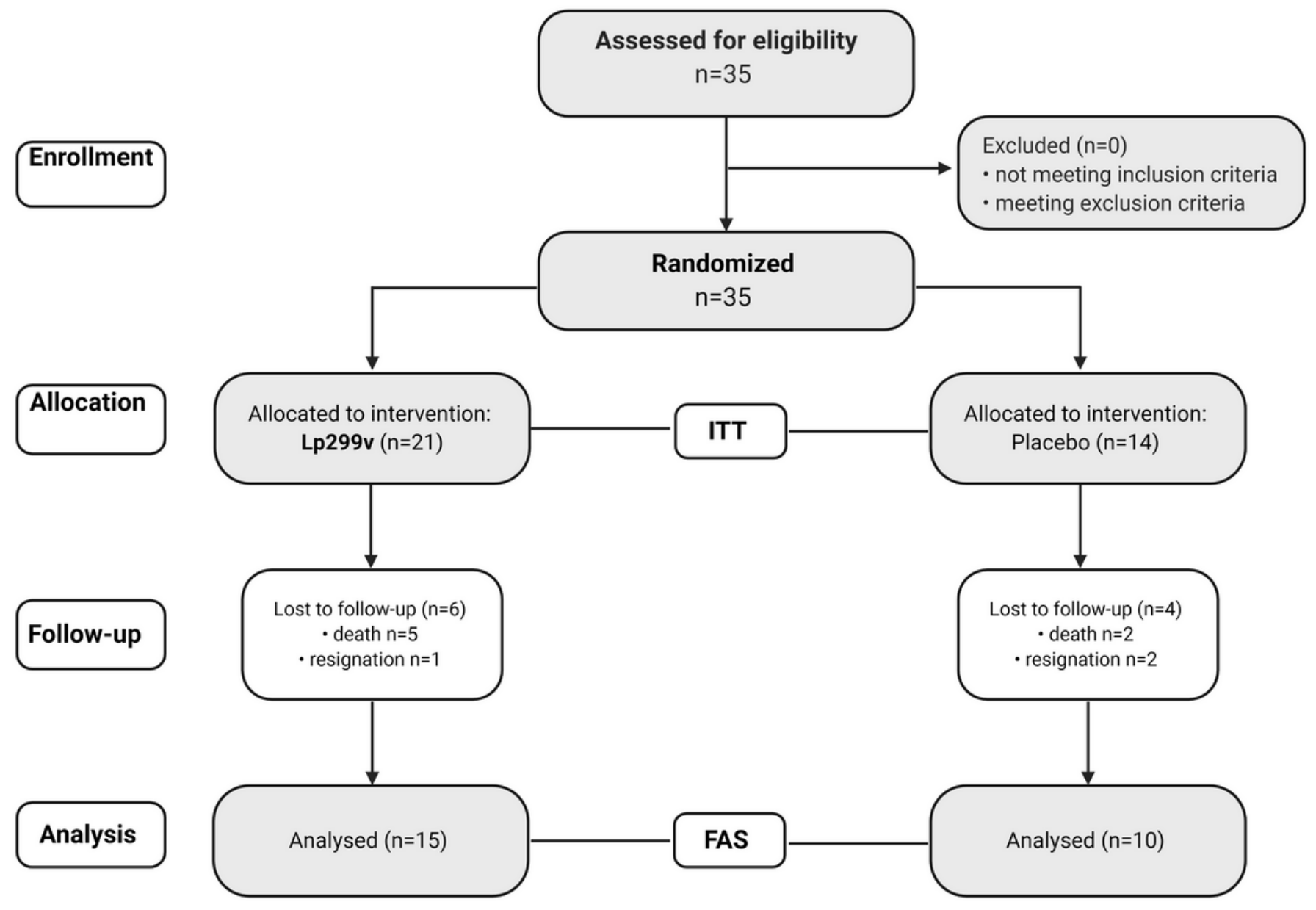

Figure 1

A flow-chart of the study design. ITT - intention to treat; FAS - full set analysis. Study products 

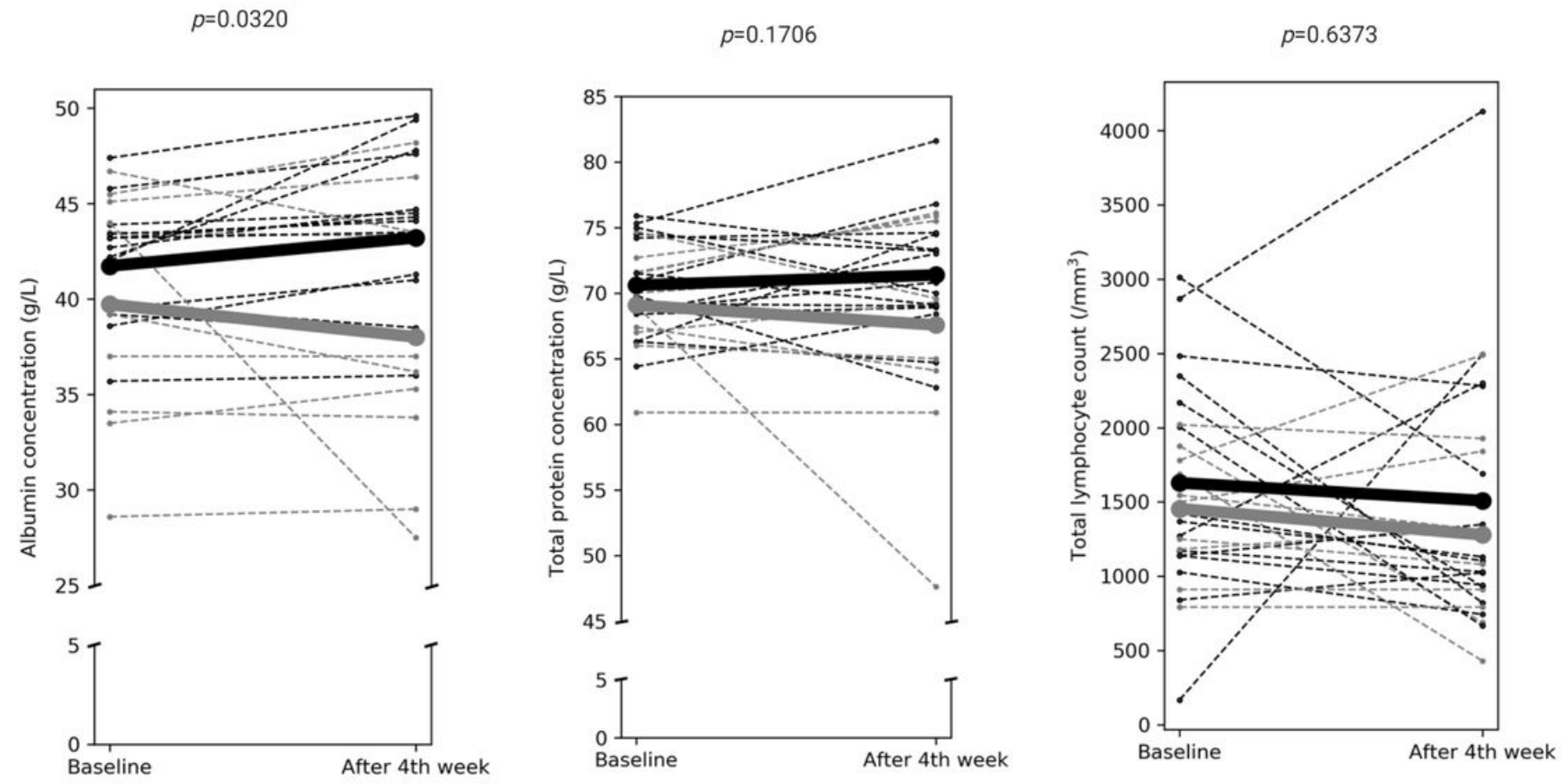

Figure 2

Changes in laboratory parameters in both groups. Dashed lines represent measurement of each patients before and after intervention. Solid lines represent mean values before and after intervention. 
A

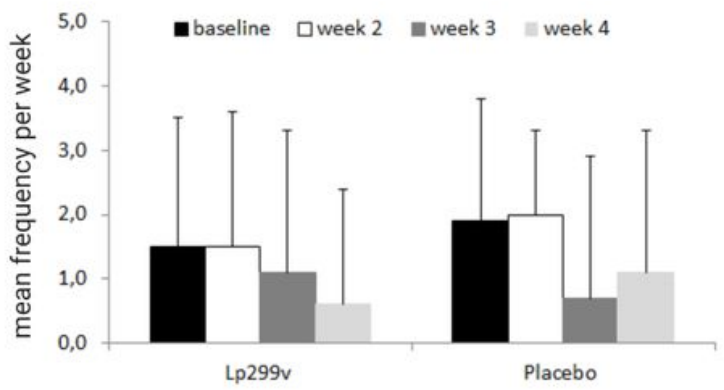

baseline: $p=0.5235$, week 2: $p=0.1741$, week 3: $p=0.5603$, week 4: $p=0.4054$
B

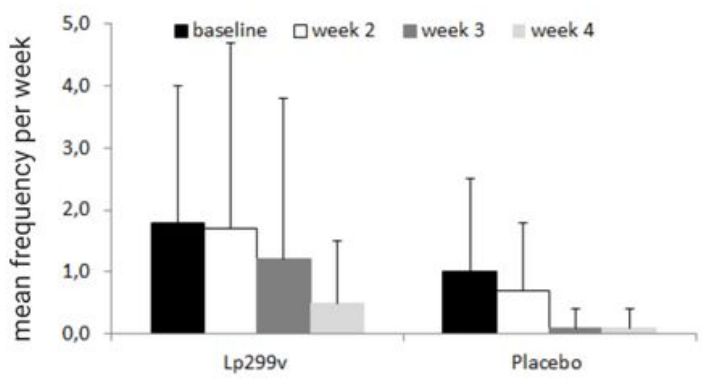

baseline: $p=0.4540$, week 2: $p=8244$, week 3: $p=0.6373$, week 4: $p=4540$

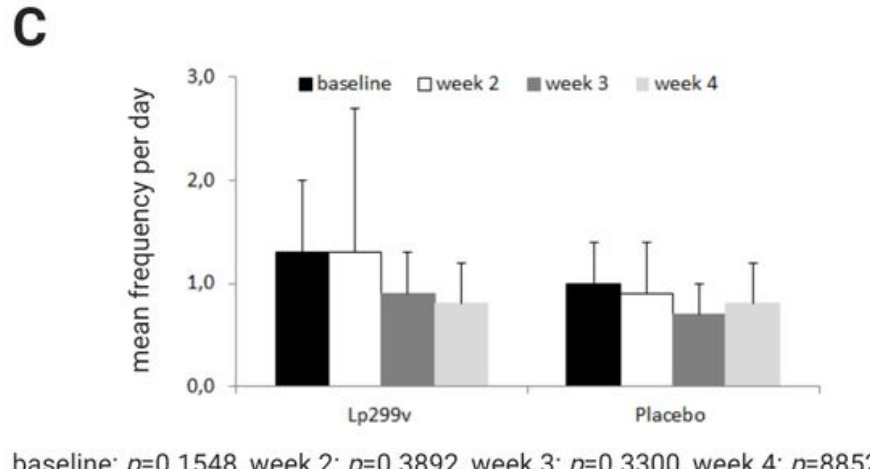

Figure 3

Changes in frequency of gastrointestinal symptoms and stools. A - vomiting; B - flatulence, C - stools. Lp299v - Lactobacillus plantarum 299v. 
A

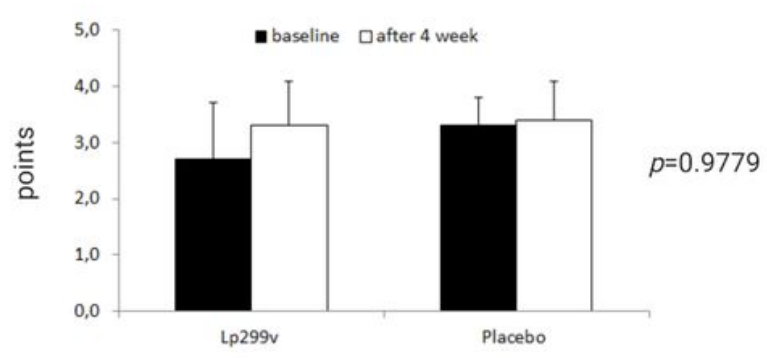

C

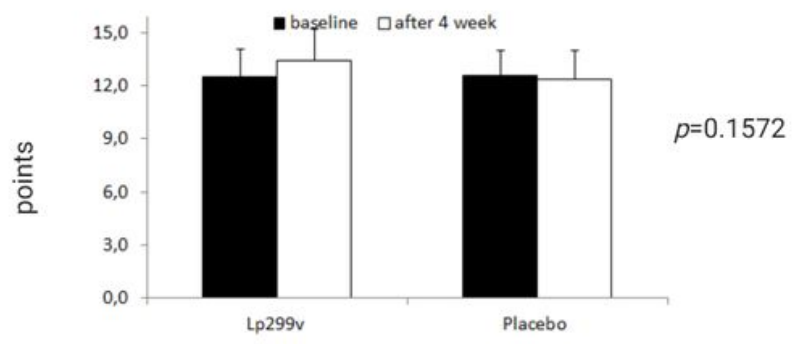

E

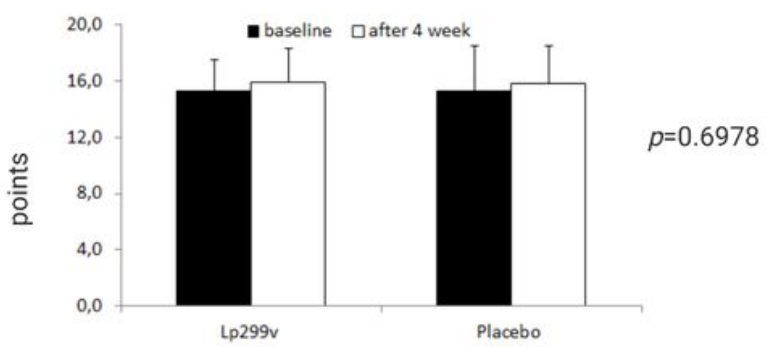

B

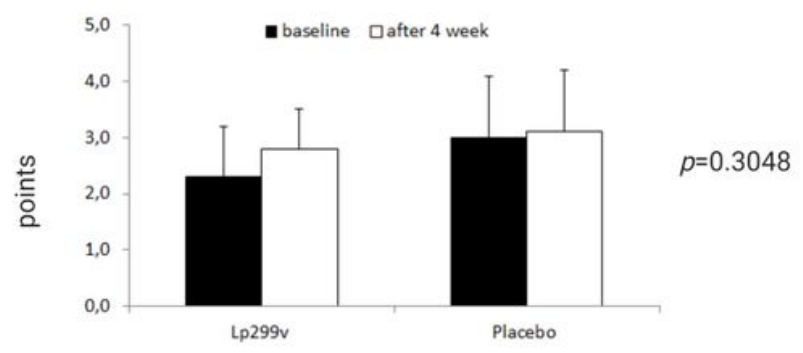

D

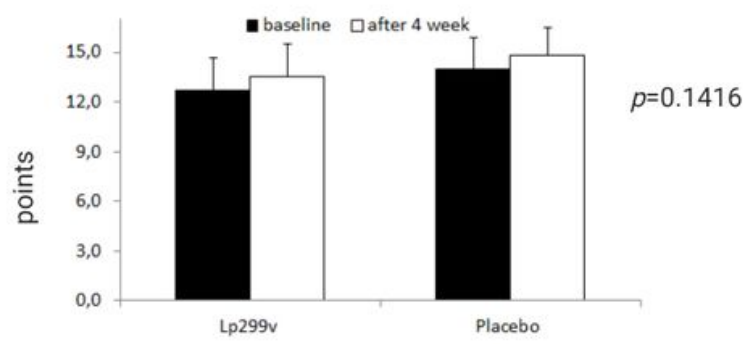

$\mathbf{F}$

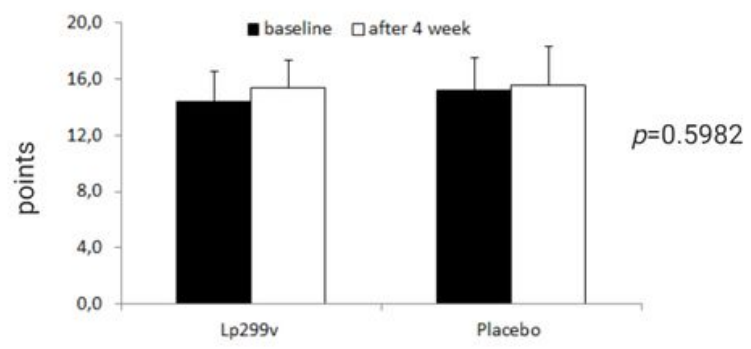

Figure 4

Changes in quality of life. A - Q1 (self-assessment of QOL); B - Q2 (self-assessment status of health); C - D1 (environmental); D - D2 (psychological); E - D3 (somatic); F - D4 (social factors). Lp299v Lactobacillus plantarum 299v. 